\title{
LOS 55 ESTADOS CON SUS RESPECTIVOS 220 ACUERDOS VIGENTES CON LA SANTA SEDE
}

\author{
Carlos Corral $^{1}$ \\ UNISCI / Universidad Complutense de Madrid (UCM)
}

\begin{abstract}
Esta nota informativa presenta una relación de los 55 estados que han firmado acuerdos aún vigentes con
\end{abstract}
Resumen: la Santa Sede y los 220 acuerdos firmados y ratificados.

Palabras clave: Santa Sede, Derecho Internacional Público, relaciones diplomáticas.

Title in English:"An Account of the 55 States and their 220 Agreements in Force with the Holy See"

\section{Abstract:}

This news brief list the 55 states having signed agreements and concordats with the Holy See, still in force, along with the 220 agreements and concordats signed and ratified.

Keywords: Holy See, International Public Law, diplomatic relations.

Copyright @ UNISCI, 2013.

Las opiniones expresadas en estos artículos son propias de sus autores, y no reflejan necesariamente la opinión de UNISCI. The views expressed in these articles are those of the authors, and do not necessarily reflect the views of UNISCI.

\footnotetext{
${ }^{1}$ Carlos Corral Salvador es Catedrático Emérito de Fuerzas Religiosas en la Sociedad Internacional de la Universidad Complutense de Madrid, e Investigador Senior de UNISCI. Sus principales líneas de investigación son la libertad religiosa, el Derecho Eclesiástico del Estado, los fundamentalismos religiosos y los Concordatos de la Santa Sede. Dirección: C/ Universidad Comillas, 7, 28049 Madrid, España.

E-mail:ccorral@res.upcomillas.es. 
En la actualidad la Santa Sede ocupa un lugar destacado en la Comunidad Internacional y su papel en los últimos años ha crecido exponencialmente hasta el punto de que tan sólo 17 Estados no tienen todavía relaciones diplomáticas con el Vaticano.

Tan es así que hoy la Santa Sede es un Sujeto de Derecho Internacional Público que goza del derecho de legación con 179 Nunciaturas Apostólicas acreditadas en otros tantos Estados y que a su vez acoge a 174 misiones en la Ciudad del Vaticano, aunque sean 69 las que no tienen su residencia en Roma por problemas de espacio. Un crecimiento así se debió, en buena parte, al impulso que le dio Juan Pablo II, al hacer pasar de 84 a 173 Estados con los que mantiene Relaciones Diplomáticas. El último Estado con quien se ha establecido las relaciones diplomáticas ha sido con el $\mathrm{Chad}^{2}$.

Pero en el fondo, dicho crecimiento obedece a dos causas. Primera, el evento trascendental del derrumbe en 1989 del bloque soviético en Europa; y segunda, la voluntad convergente de los Estados liberados del yugo moscovita de querer normalizar las relaciones que, otrora, entre ambas guerras mundiales habían mantenido las relaciones diplomáticas con la Santa Sede.

Como consecuencia se ha producido una saga cada vez más larga de Acuerdos (llámense Concordatos, Convenios o Canje de Notas) que al presente suman, según nuestro cómputo, nada menos que 219.

Todo ello se hace realidad gracias a la política internacional convergente llevada a cabo por la Santa Sede y a la consecuente expansión creciente de los Acuerdos con los Estados por los cinco continentes.

\section{I.- El aumento creciente de los Acuerdos con los Estados es el resultado de una política internacional convergente de la Santa Sede}

Para ello, se han diseñado y se están llevando a término tres clases de políticas.

$1^{a}$ / una política de consolidación y renovación de los acuerdos concordatarios con 55 Estados:

A/ con los 3 países germánicos Alemania (y con 16 de sus Länder), Austria y Suiza (y con 6 de sus Cantones).

B/ con los 8 Estados latinos: Andorra, España, Francia, Italia, Malta, Mónaco (Principado de), Portugal y San Marino.

C/ con los 11 Estados concordatarios de Hispano-América: Argentina, Bolivia, Brasil, Colombia, Ecuador, El Salvador, Haití, Paraguay, Perú, República Dominicana y Venezuela.

\footnotetext{
${ }^{2}$ Referencia bibliográfica: Corral, Carlos (2009): Derecho Internacional concordatario, Madrid, Biblioteca de Autores Cristianos; Santos J.L. y Corral, C. (2006): Acuerdos entre la Santa Sede y los Estados, Versión española de los textos, Madrid, B.A.C.
} 
2\% una política de recuperación de la vía concordataria con los Estados de Europa Oriental liberados del comunismo:

A/ con los 5 bálticos (Estonia, Letonia, Lituania, Polonia y Suecia).

B/ con los 8 danubianos (Albania, Bosnia-Herzegovina, Croacia, Eslovaquia, Eslovenia, Hungría, Montenegro); [incluida Chequia, con convenio firmado si bien no ratificado].

\section{3\% una política de expansión de los acuerdos concordatarios a otros Estados mediante:}

A/ la accesión de 4 Micro-Estados a la vía concordataria: Andorra, Malta, Mónaco y San Marino.

B/ la incorporación de 11 Estados africanos: desde el mediterráneo al interior: Burundi, Camerún, Costa de Marfil, Chad, Gabón, Guinea Ecuatorial, Liga de Estados Árabes, Marruecos, Mozambique, Organización de la Unidad Africana (OUA =U.A) y Túnez.

C/ la adhesión de 8 Estados del Próximo y Lejano Oriente: Azerbaiyán, China/Taiwán, Filipinas, [Georgia] Israel, Kazajstán, Palestina [O.L.P.] y Vietnam.

Pero con una peculiaridad muy adecuada para el ordenamiento concordatario en su conjunto. Consiste en que, por un lado, las cuestiones ordinarias comunes vinieron tratándose con cada vez más perfectas regulaciones; y en que, por otro, las cuestiones surgidas específicamente a los Estados recién independizados recibieron las correspondientes soluciones novedosas en los recientes Convenios con la Santa Sede. Un estudio tan complejo a nivel comparado de varios años es lo que ha constituido el objeto de nuestro reciente libro Derecho Internacional concordatario (Madrid 2009).

\section{II.- La creciente expansión transnacional de los Convenios de los Estados con la Santa Sede}

Dichos Convenios, al presente, ya no conocen fronteras ni estatales ni continentales. En un principio, originarios de las naciones europeas, comenzaron a pasar desde el siglo XIX de Europa a América y, después — si bien todavía en forma limitada— a África y Asia. Teniendo en cuenta, por un lado, la cultura y la lengua, y, por otro, los vínculos de vecindad, se podrían agrupar los Concordatos vigentes en cuatro grupos de conformidad con los Estados: germánicos, latino-europeos, latinoamericanos y afroasiáticos.

Al presente, nada menos que 55 Estados [contando por ahora a Palestina (O.L.P.), a la Liga de Estados Árabes, a la Organización de la Unidad Africana (O.U.A) y a la Unión Europea, aun cuando no sean Estados], e incluyendo a Chequia y Georgia con sendos Convenios firmados si bien no ratificados] mantienen en vigor 219 Acuerdos o Convenios con la Santa Sede, de los que 36 son de carácter general y 183 de carácter parcial (o sectorial).

A los 55 Estados habría que añadir otras entidades territoriales que — sin ser Estados, tienen personalidad internacional, si bien limitada — como las 16 Regiones alemanas (Länder) 
y los 6 Cantones suizos (dando lugar a un total de 87 sujetos internacionales que se interesan o están interesados en mantener alguna forma de acuerdo con la Sede Apostólica.

He aquí los 55 Estados concordatarios [incluyendo a la Liga de Estados Árabes, la Organización de la Unidad Africana (O.U.A) y la Unión Europea].

Albania, Alemania (y 16 Länder: Baden-Württemberg, Baja Sajonia, Baviera, Brandeburgo, Mecklenburgo-Pomerania, Prusia, Renania del Norte Westfalia, RenaniaPalatinado, Sarre, Sajonia, Sajonia-Anhalt, Sarre, Schleswig-Holstein y Turingia, más las 2 Ciudades Hanseáticas de Bremen y Hamburgo), Andorra, Argentina, Austria, Azerbaiyán, Bolivia, Bosnia-Herzegovina, Brasil, Burundi, Camerún, Colombia, Costa de Marfil, Croacia, [Chequia], China/Taiwán, Ecuador, El Salvador, Eslovaquia, Eslovenia, España, Estonia, Filipinas, Francia, Gabón, [Georgia], Guinea Ecuatorial, Marruecos, Haití, Hungría, Israel, Italia, Kazajstán, Letonia, Liga de Estados Árabes, Lituania, Malta, Marruecos, Mónaco (Principado de), Montenegro, Mozambique, Organización de la Unidad Africana (O.U.A), Palestina (Organización para la Liberación de Palestina), Paraguay, Perú, Polonia, Portugal, República Dominicana, San Marino, Suecia, Suiza (y 6 cantones: Argovia-Turgovia, Berna, Friburgo, Lucerna, San Gal y Ticino), Túnez, Unión Europea, Venezuela, Vietnam.

De dichos 55 Estados, 37 Estados tienen una regulación complexiva por medio del correspondiente Acuerdo/Convenio de carácter general, (de los cuales, 6 la hacen adoptando la formalidad solemne de un Concordato — que se señalan con un *):

Albania, *Alemania, Argentina, *Austria, Bosnia-Herzegovina, *Colombia, [Chequia], Chad, Ecuador, Eslovaquia, Eslovenia, Estonia, España, Filipinas, Francia, Gabón, [Georgia], Haití, Hungría, Israel, Italia, Kazajstán, Letonia, Lituania, Marruecos, Mónaco (Principado de), Montenegro, Palestina (Organización para la Liberación de Palestina), Organización para la Unidad Africana (O.U.A = U.A), Perú, *Polonia, *Portugal, *República Dominicana, San Marino, Suecia, Suiza, Túnez y Venezuela. De ellos, 6 Estados tienen dicha regulación complexita, pero sirviéndose de un acervo de acuerdos sectoriales, como Croacia, Eslovaquia, España, Francia, Lituania y Malta.

Y 10 Estados tienen una regulación sólo parcial mediante un acuerdo sectorial: Bolivia, Camerún, Costa de Marfil, El Salvador, Filipinas, Paraguay, Suecia, Suiza, Unión Europea, Vietnam.

De los 16 Länder concordatarios, 8 tienen una regulación complexiva, de los cuales, 4 (Baden-Wüttemberg, Baja Sajonia, Baviera, [antes Prusia], mediante un Concordato; los otros 8 (Brandeburgo, Mecklenburgo-Pomerania, Sajonia, Sajonia-Anhalt, SchleswihHolsstein y Turingia más Bremen y Hamburgo) mediante el correspondiente convenio de carácter general; en cambio, tienen una regulación sectorial, 3: Renania del Norte-Westfalia, Renania-Palatinado y el Sarre.

No está de más llamar la atención sobre el in crescendo que vienen experimentando los convenios concordatarios que se fueron estipulando sin pausa durante los tres últimos pontificados de Juan Pablo II, Benedicto XVI y Francisco I. En efecto, a los precedentes Estados concordatarios, se han ido sumando 30 nuevos Estados: Azerbaiyán, Brasil, Burundi, Camerún, Chad, Chequia, China/Taiwan, Costa de Marfil, Eslovaquia, Eslovenia, Estonia, Gabón, Guinea Ecuatorial, Hungría, Israel, Kazajstán, Letonia, Liga de Estados Árabes, Lituania, Malta, Marruecos, Mozambique, O.U.A., Palestina, Polonia, San Marino, Suecia, Unión Europea, Vietnam, y otros seis Länder: Brandeburgo, Meckenburgo-Pomerania, 
Sajonia, Sajonia-Anhalt, Schleswig-Holstein y Turingia, más las Ciudades Hanseáticas Libres de Bremen y Hamburgo).

\section{III.- La universalidad religioso-política de los Convenios de los Estados con la Santa Sede}

Mientras en épocas pasadas los Concordatos se concluían en gran parte con Estados católicos o de gran minoría católica, hoy en día ocurre exactamente al revés. En efecto, de los 55 Estados que han celebrado Concordatos.

- 6 son confesionales: 4 católicos: Haití, Malta, Mónaco y República Dominicana; y 3 musulmanes: Azerbaiyán, Túnez y Marruecos.

- 49 son separacionistas: Albania, Alemania, Andorra, Argentina, Austria, Bolivia, BosniaHerzegovina, Brasil, Burundi, Camerún, Chad, [Chequia], China/Taiwán, Colombia, Costa de Marfil, Croacia, Ecuador, El Salvador, Eslovaquia, Eslovenia, España, Estonia, Filipinas, Francia, Gabón, [Georgia], Guinea Ecuatorial, Hungría, Israel, Italia, Kazajstán, Letonia, Lituania, Mozambique, Palestina (O.L.P.), Paraguay, Perú, Polonia, Portugal, O.U.A (Organización de la Unión Africana = hoy U.A.), San Marino, Suecia, Suiza, Unión Europea, Venezuela, y Vietnam.

- Deben contabilizarse, además, los 16 Länder de Alemania Federa y los 6 Cantones de Suiza.

En conclusión, si el final de la I Guerra mundial comportó, para el ámbito de las relaciones diplomáticas y convencionales (aquí, las concordatarias), un nuevo diseño ante unas nuevas realidades que llevó a la configuración de una "nueva era de concordatos" [de la que siguen siendo modelos en parte todavía vigentes los de Alemania 1933, Austria 1932 e Italia 1929, más Francia de 1801 para Alsacia-Lorena], el derrumbamiento del bloque soviético ha comportando, si no un nuevo diseño, sí, al menos, un nuevo impulso renovador. Así ha ocurrido al presente, a nuestro parecer; y tal fue la política seguida hasta ahora por Pío XII, Paulo VI, Juan Pablo II, actualmente por Benedicto XVI — quien comenzó ya por el importante Acuerdo de 13-11-2009 con el inmenso Brasil, tan multiétnico como multicultural-y Francisco I.

IV.- RELACIÓN ALFABÉTICA de los 55 Estados con sus respectivos 220 Concordatos y/o Acuerdos (Canjes, Convenciones, Protocolos) vigentes.

De los 220, al presente están publicados 212 con sus originales, traducciones e introducciones en 5 Tomos I a V por C. CORRAL con otros Profesores. Los Tomos I y II por C. Corral y J. Gz. DE CARVAJAL (dirs.), Concordatos vigentes, textos originales, traducciones e introducciones, (Madrid, Fundación Universitaria Española 1981); el Tomo III, por C. CORRAL y S. PETSCHEN, Concordatos vigentes, textos originales, traducciones e introducciones (1981-1995), (Madrid, Fundación Universitaria Española 1996); el Tomo IV por C. CORRAL y S. PETSCHEN, Tratados internacionales (1996-2003), (Madrid, Universidad Pontificia Comillas 2004); el Tomo V por C. CorRaL y J. L. Santos, Tratados internacionales 
2003-2012) de la Santa Sede con los Estados. Concordatos vigentes, (Madrid, Universidad Pontificia Comillas, E.Book 2012).

Entre los 55 Estados, aquí elencados, se incluyen la Liga Árabe, la Unión Europea y la Unión Africana. A los 212 Concordatos publicados en los Tomos I a V, se añaden aquí 8 más posteriores-que se reenvían a un futuro tomo VI seguido de nn. Además se elencan los 16 Länder y los 6 Cantone s=22 Regiones.
ALBANIA
(1)- I.- Acuerdo [general] de 23-3-2002
2)- II.- Acuerdo, de 3-12-2007, sobre algunas cuestiones económicas y tributarias

\section{ALEMANIA}

-(3)- I. Concordato de 20 julio 1933

- Protocolo Final de 20 julio 1933

-(4)- II Anejo secreto de 20 julio 1933 al Concordato del Reich (remisión)

-(5)- III Canje de Notas de 16/17 julio sobre la interpretación del artículo 26

del Concordato del Reich (remisión)

-(6)- IV Estatutos de la asistencia religiosa a las Fuerzas Armadas de la

República Federal de Alemania de 31 julio 1965 (remisión)

-(7)- V Nota verbal de la Nunciatura Apostólica en Alemania de 22 febrero

1966, sobre la interpretación del artículo 15, párrafo 2, parágrafo I

del Concordato del Reich (remisión)

\section{ALEMANIA FEDERAL}

-(8)- VI Acuerdo mediante Canje de Notas de 10/16 enero 1990, sobre la disciplina y los Estatutos del Ordinariato Castrense 
ANHALT

-(9)- I. Acuerdo de 4 enero 1932, sobre el reembolso de los costes de pensión a favor de las escuelas católicas, (remisión)

-(10)- II. Acuerdo de 4 enero 1932, sobre la dotación del Estado a las comunidades parroquiales católicas

\section{BADEN-WÜRTTENBERG}

-(11)- Concordato de 12 octubre 1932

- Protocolo Final de 12 octubre 1932

- $\quad$ Protocolo Adicional de 7/10 noviembre 1932

\section{BAJA SAJONIA}

-(12)- I. Concordato de 26 febrero 1965

- $\quad$ Anejo al Concordato de 26 febrero 1965

- $\quad$ Protocolo de 26 febrero 1965, sobre la negociación del Concordato

- $\quad$ Protocolo de 26 febrero 1965, de la sesión de clausura

-(13)- II Convenio de 21 mayo 1973, para la modificación del Concordato

-(14)- III. Convenio de 8 mayo 1989, para la modificación del Concordato de 26 de febrero de 1965

-(15)- IV. Convenio de 29 de octubre 1993, para la modificación del Concordato de 26 de febrero de 1965

-(16)- V. Acuerdo, de 6-4-2010, para introducir algunas modificaciones en el Concordato.

\section{BAVIERA}

-(17)- I. Concordato de 29 marzo 1924 I

-(18)- Acuerdos adicionales de 1927/28 y 16 julio 1931 al Concordato (remisión)............ I

-(19)- Acuerdo administrativo de 29/30 agosto 1958 (remisión)........................................ 
-(20)- II. Convenio de 2 septiembre 1966, sobre la Facultad de Teología Católica de la Universidad de Regensburg. ..I

-(21)- III. Convenio de 2 septiembre 1966, sobre la supresión de la Escuela Superior de Filosofía y Teología de Freising y la formación científica de los estudiantes de Teología Católica en la Universidad de Munich. ..I

-(22)- IV. Convenio de 17 septiembre 1970, sobre la Facultad de Teología Católica de la Universidad de Augsburgo. ..I

- $\quad$ Protocolo Final de 27 septiembre 1970..............................................................

-(23)- V. Convenio de 4 septiembre 1974, para modificar y completar el Concordato bávaro

- $\quad$ Protocolo Final de 4 septiembre 1974 ..............................................................

- $\quad$ Canje de Notas de 4 septiembre 1974, entre el Nuncio Apostólico en Alemania

y el Ministro Presidente de Baviera (remisión)..............................................................

-(24)- VI. Convenio de 7 julio 1978, para la modificación del Concordato .........................

Protocolo Final de 7 julio 1978 ...........................................................................

-(25)- VII. Convenio de 8 junio 1988, por el que se introducen algunas modificaciones en el Concordato con Baviera de 29 marzo 1924 III

Protocolo Final de 8 de junio 1988 III

-(26)- Protocolo Adicional, de 19-1-2007, al Concordato. $\mathrm{V}$

BRANDEBURGO.

-(27)- Convenio — General—de 12-11-003... ..V

\section{BREMEN}

-(28)- Convenio — general— de 21-11-2003 V

\section{HAMBURGO}

-(29)- -I. Convenio — general— de 29-11-2005....................V

Introducción......................................................

-(30)- - II. Convenio para la erección de un centro de formación para la Teología...V 


\section{MECKLENBURGO-POMERANIA ANTERIOR}

-(31)- Acuerdo [general] de 18 octubre 1997. IV

PRUSIA

-(32)- Concordato de 14 junio 1929 I

Protocolo Final I

Canje de Notas de 5/6 agosto 1929, entre el Nuncio Apostólico en Berlín y el

Ministro Presidente de Prusia. I

- $\quad$ Canje de Notas de 4 agosto 1933, entre el Nuncio Apostólico en Berlín y el

Ministro prusiano de Educación (remisión) .I

- $\quad$ Canje de Notas de 17 julio/3 agosto 1933, entre el Ministro Presidente de

Prusia y el Cardenal Secretario de Estado (remisión) I

\section{RENANIA-PALATINADO}

-(33)- I. Convenio de 29 abril 1969, para completar y modificar las disposiciones del

Concordato en Renania-Palatinado I

Protocolo Final de 29 abril 1969. I

-(34)- I. Convenio de 15 mayo 1973, sobre cuestiones concernientes a las escuelas y al perfeccionamiento y formación continuada de los maestros .I

Protocolo Final de 15 mayo 1973 I

\section{RENANIA DEL NORTE-WESTFALIA}

-(35)- I. Convenio de 19 diciembre 1956 I

-(36)- I. Canje de Notas de 20/29 diciembre 1967, sobre el Departamento de Teología

Católica en la Universidad del Ruhr en Bochum (remisión) I

-(37)- II. Canje de Notas de 21/22 abril 1969, sobre cuestiones relativas a la reforma de la formación de los maestros (remisión) .I 


\section{SAJONIA}

-(38)- Acuerdo [general] de 2 abril 1996 IV

\section{SAJONIA-ANHALT}

-(39)- Acuerdo [general] de 15 enero 1998 IV

Acuerdo [general] de 2 abril 1996. IV

\section{SARRE}

-(40- I. Convenio de 9 abril 1968, sobre la erección de una cátedra de Teología Católica en la Universidad del Sarre.... .I

- $\quad$ Protocolo Adicional de 9 abril 1968 I

-(41)- II. Convenio de 12 noviembre 1969, sobre la formación de maestros I

Protocolo Adicional de 12 noviembre 1969. I

III Convenio de 21 febrero 1975, sobre las escuelas privadas dirigidas por la

Iglesia Católica... I

- $\quad$ Protocolo Adicional de 21 febrero 1975 .............................................................. I

-(42)- IV. Convenio de 12 febrero 1985 ................................................................... IV

Protocolo Adicional de 12 febrero 1985 ............................................................ III

-(43)- V. Convenio de 19 de septiembre de 2001 para modificar el Convenio de ejecución sobre escuelas de titularidad de la Iglesia.... IV

SCHLESWIG-HOLSTEIN

-(44)- Acuerdo - General— de 12-1.2009. ... V.

\section{TURINGIA}

-(45)- I. Acuerdo [general] de 11 junio 1997 IV

-(46)- II. Convenio de 16 diciembre 2003, sobre Facultad de Teología Católica en la Universidad de Erfurt. ..IV 276 


\section{VARIAS REGIONES NUEVAS}

-(47)- I. Convenio de 4 mayo 1994, con Brandeburgo y el Estado Libre de Sajonia sobre

la erección de la diócesis de Görlitz .III

-(48)- II. Convenio de 13 abril 1994, con Sajonia-Anhalt, Brandeburgo y el Estado Libre de.Sajonia sobre la erección de la diócesis de Magdeburgo..........III 173

-(49)- III. Convenio de 14 junio 1994, con el Estado libre de Turingia sobre la erección de la diócesis de Erfurt III

-(50)- IV. Convenio de 22 septiembre 1994, con la Ciudad Libre Hanseática de Hamburgo, la Región de Mecklenburgo-Pomerania Anterior y la Región de Schleswig-Holstein sobre la erección de la Archidiócesis y Provincia Eclesiástica de Hamburgo. .III

\section{ANDORRA}

-(51)- Acuerdo-General- de 17-3-2008 $\mathrm{V}$

\section{ARGENTINA}

-(52)- I. Acuerdo de 28 junio 1957, sobre Jurisdicción Castrense y Asistencia

religiosa de las Fuerzas Armadas II

-(53)- II. Acuerdo [general] de 10 octubre 1966, II

-(54)- III. Canje de Notas de 21 abril 199. .IV

\section{AUSTRIA}

I. -(55)- I. Concordato de 5 junio 1933 .I

Protocolo Adicional, de 5 junio 1933 I

II. -(56)- II. Convenio de 23 junio 1960, para la regulación de las relaciones patrimoniales

III. -(57)- III. Convenio de 23 junio 1960, relativo a la erección en diócesis de la Administración Apostólica de Burgenland 
IV. -(58)- IV. Convenio de 9 julio 1962, para regular las cuestiones referentes a la organización escolar.

- $\quad$ Protocolo Final de 9 julio 1962

-(59)- V, Convenio de 7 julio 1964, relativo a la erección en diócesis de la

Administración Apostólica de Innsbruck-Feldkirch I

-(60)- VI. Convenio de 7 octubre 1968, relativo a la erección en diócesis de Feldkirch.... I

-(61)- VIII. Convenio Adicional de 8 marzo 1971, para regular las cuestiones relativas a la organización escolar

-(62)- IX Sexto Acuerdo Adicional, de 10-3-2009.

\section{AZERBAIYÁN}

-(63)- Acuerdo de 29-4-2011 $\mathrm{V}$

\section{BOLIVIA}

-(64)- I. Convenio de 4 diciembre 1957, sobre las Misiones

II

- $\quad$ Apéndice al Convenio sobre las Misiones (art. I n.3) II

-(65)- I. Acuerdo de 29 noviembre 1958, sobre Jurisdicción eclesiástica castrense y asistencia religiosa de las Fuerzas armadas

-(66)- III. Acuerdo de 1 diciembre 1986, sobre asistencia religiosa a las Fuerzas Armadas y Fuerzas de Policía. III

-(67)- IV. Acuerdo de 20 de agosto de 2009 sobre 20 de agosto de 2009 entre el Gobierno boliviano .VI nn.

\section{BOSNIA-HERZOGOVIA}

-(68)- I. Acuerdo básico de 19-4-2006.................................V

-(69)- II. Protocolo de 29-9-2006.....................................

** Introducción......................................................

-(70)- III. Acuerdo, de 8.4.2010, sobre Asistencia religiosa a los fieles católicos de las Fuerzas Armadas. $\mathrm{V}$ 


\section{BRASIL}

-(71)- I. Acuerdo de 23 octubre 1990, sobre Asistencia Religiosa a las Fuerzas ArmadasIII

-(72)- Acuerdo - General- de 13-11-2008. V

\section{BURUNDI}

-(73)- Acuerdo Marco sobre el estatuto jurídico de la Iglesia, de 8-11-2012...VI

\section{CAMERÚN}

-(23)- I. Acuerdo de 5 julio 1989, relativo a la sede del Instituto Católico de Yaoundé ... IV

-(74)- II. Acuerdo de 17 agosto 1995, relativo al reconocimiento de los diplomas expedidos por el Instituto Católico de Yaoundé. IV

\section{CHAD}

-(76)- Acuerdo de 3 de noviembre de 2013 sobre "Estatuto jurídico de la Iglesia Católica en el Chad. .VI

\section{CHECOSLOVAQUIA}

-(76/B)- Acuerdo de 27 febrero 1973[abrogado] II

\section{CHEQUIA}

-(77)- Acuerdo, de 25-7-2002, sobre regulación de las relaciones recíprocas[no aprob.VII 04]. ..V

\section{CHINA/TAIWAN}

-(78)- Acuerdo cultural, 17 de diciembre de 2012 VI 


\section{COLOMBIA}

-(79)- I. Concordato de 12 julio 1973, entre la República de Colombia y la Santa Sede ..II

- $\quad$ Protocolo Final de 12 Julio 1973 ......................................................................

-(80)- - Declaración de 2 julio 1975, en el momento del canje de los instrumentos de

ratificación del Concordato

-(81)- II. Acuerdo de 20 noviembre 1992 [no ratificado], con el cual se introducen modificaciones al Concordato del 12 de julio de 197. IV

\section{COSTA DE MARFIL}

-(82)- I. Convenio de 20 mayo 1992, concerniente a la "Fundación Internacional

Nuestra Señora de la Paz" de Yamasucro III

-(83)- II. Convenio de 14 agosto 1989, concerniente a las estaciones de radiodifusión.....IV

\section{CROACIA}

-(84)- I. Acuerdo de 19 diciembre 1996, sobre cuestiones jurídicas IV

-(85)- II. Acuerdo de 19 diciembre 1996, sobre la colaboración en el campo educativo y cultural IV

-(86)- III. Acuerdo de 19 diciembre 1996, sobre la asistencia religiosa a los fieles de las Fuerzas Armadas y de la Policía de Croacia ..................................................................

-(87)- IV. Acuerdo de 9 octubre 1998, sobre cuestiones económicas. IV

\section{ECUADOR}

-(88)- I. "Modus vivendi” [general] de 24 julio 1937 ...........................................................

-(89)- II. Convenio Adicional de 24 julio 1937 ...................................................................

*Introducción: el Ordinariado Castrense...............................................................................III 
-(89/B)- III. Acuerdo de 3 agosto 1978, sobre la Asistencia Religiosa a las Fuerzas Armadas y a la Policía Nacional.

-(90)- IV. Canje de Notas de 27 enero/6 julio 1982

\section{EL SALVADOR}

-(91)- Convenio de 11 marzo 1968, sobre jurisdicción eclesiástica castrense y asistencia religiosa de la Fuerza Armada y Cuerpos de Seguridad .II

\section{ESLOVAQUIA}

-(92)- I. Acuerdo base de 24 noviembre 2000 IV

-(93)- II. Acuerdo de 21 de agosto de 2002 sobre la asistencia religiosa a los fieles católicos en las Fuerzas Armadas y en los Cuerpos rmados IV

**Introducción V

-(94)- III. Acuerdo, de 13-5-2004, sobre la educación y enseñanza religiosa...V

\section{ESLOVENIA}

-(95)- Acuerdo de 11-12- diciembre 2001, sobre cuestiones jurídicas IV

\section{ESPAÑA}

-(96)- I. Acuerdo de 28 julio 1976 [Básico] ...I

-(97)- II. Acuerdo de 3 enero 1979, sobre Asuntos Jurídicos. ............................................II

Protocolo Final de 3 enero 1979 .......................................................................... II

-(98)- III. Acuerdo de 3 enero 1979, sobre la asistencia religiosa a las Fuerzas Armadas y el servicio militar de clérigos y religiosos II

Protocolo Final de 3 enero 1979 II

Anexo I II

Anexo II II

-(99)- IV. Acuerdo de 3 enero 1979, sobre enseñanza y Asuntos Culturales. .....................II

Disposiciones transitorias. II 
- $\quad$ Protocolo Final de 3 enero 1979 ...........................................................................

-(100)- V. Acuerdo de 3 enero 1979, sobre Asuntos Económicos......................................II

- $\quad$ Protocolo Adicional de 3 enero 1979 ................................................................

-(101)- VI. Acuerdo de 10 octubre 1980, acerca de la aplicación del Impuesto sobre

sociedades a las Entidades eclesiásticas.

-(102)- VII. Acuerdo de 21 diciembre 1994, sobre asuntos de interés común en Tierra Santa. .III

-(103)- VIII. Canje de Notas, de 21/22-12-200. .. $V$

\section{ESTONIA}

-(104)- Canje de Notas de 23 diciembre 1998/15 febrero 1999 [general, CR. 12 III 99]..IV

\section{FILIPINAS}

-(105)- I. Intercambio de notas de 20 septiembre 1951/28 marzo 1952, relativo a la erección del Vicariato castrense en las Fuerzas Armadas .II

-(106)- II. Acuerdo, de 1-4-2007, sobre bienes culturales. V

\section{FRANCIA Y ALSACIA-LORENA}

*Introducción, por C. Corral II

-(107)- I. Convención de 15 julio 1801, entre su Santidad Pio VII y el Gobierno francés II

-(108)- II. Acuerdo de 5 diciembre 1902, sobre la erección de una Facultad de Teología Católica en la Universidad "Kaiser Wilhelm" de Strasburgo

-(109)- II. Acuerdo de mayo 1921, sobre el nombramiento de los Obispos en Francia ....II

-(110)- IV. Canje de notas de 7/13 mayo 1923, sobre la validez del proyecto de Estatutos de Asociaciones diocesanas

a) Nota de Mons. Cerretti, Nuncio Apostólico a M. Poincaré, Presidente del Consejo de Ministros, de 7 mayo 1923 II

b) Proyecto de Estatutos de "Asociaciones diocesanas" II

c) Nota de M. Poincaré, Presidente del Consejo de Ministros en respuesta a la del 
Nuncio Apostólico, de 13 mayo 1923.

d) Extracto del registro de las deliberaciones del Consejo de Estado

de 13 diciembre 1923.

-(111)- V. Canje de notas de 16/17 noviembre 1923, entre el Nuncio Apostólico en Francia y el Presidente del Consejo de Ministros, Ministro de Asuntos Exteriores,

confirmando el Convenio de 5 diciembre 1902

a) Nota de 16 noviembre 1923, de la Nunciatura Apostólica de Francia al Presidente del Consejo, Ministro de Asuntos Exteriores. II

b) Nota de 17 noviembre 1923, del Sr. Ministro de Asuntos Exteriores a Mons.

Cerretti, Nuncio Apostólico II

-(112)- VI. Acuerdo de 4 diciembre 1926, sobre los honores litúrgicos en los países en los que se ejerce el protectorado religioso francés. .II

-(113)- VII. Acuerdo de 4 diciembre 1926, sobre los honores litúrgicos en los países en donde las capitulaciones son abrogadas o no se aplican .II

-(114)- VIII. Convenio de 25 mayo 1974, relativo al Centro Autónomo de Enseñanza de Pedagogía Religiosa del la Universidad de Metz. .II

IX. "Santa Trinitá dei Monti" IV

-(115)- 1. Convención diplomática de 14 mayo 1828, para la conversión del Convento de los mínimos de la Trinitá dei Monti en casa de educación bajo la Dirección de las

Damas Francesas del Sagrado Corazó. IV

-(116)- 2. Convención diplomática complementaria de 8 septiembre de 1828...IV

-(117)- 3. Acuerdo Adicional de 4 mayo de 1974. IV

-(118)- 4. Acuerdo Adicional de 21 enero 1999

-(119)- X. Canje de Notas, de 12-11-2001, sobre el Estatuto de las Obras Misionales Pontificias. ..V

-(120)- XI. Avenant, Protocolo Adicional, de 12-7-2005, relativo a Trinità in Monte. . V

-(121)- XII. Acuerdo, de 18-12-2008, sobre el reconocimiento de grados y diplomas en la enseñanza superior. ... V

\section{GABÓN}

-(122)- I. cuerdo Marco de 12 diciembre 1997 IV 
-(123)- II. Acuerdo de 26 de julio de 2001 .IV

\section{GUINEA ECUATORIAL}

-(124)- Acuerdo de 13 de octubre de 2012. VI

\section{HAITÍ}

-(125)- I. Concordato de 28 marzo 1860, entre el Papa Pio IX y la República de Haití.... II

a) Carta del Ministro Plenipotenciario de Haití al Cardenal Antonelli, de 28 marzo 1860.. II

b) Nota respuesta del Cardenal Antonelli, de 28 marzo 1860 II

c) . Convenio de 12 marzo 1861, sobre circunscripciones eclesiásticas .II

-(127)- III. Convenio [adicional] de 17 junio 1862,

II

-(128)- IV. Convenio de 25 enero 1940, entre la Santa Sede y la República de Haití sobre los bienes de la Iglesia Católica en Haití y sobre la organización y la administración de las fabricas parroquiales II

-(129)- V. Protocolo de 15 agosto 1966, entre los Plenipotenciarios de Su Santidad el Papa Pablo VI y los Plenipotenciarios de su Excia. el Dr. Francois Duvalier, President à Vie de la République d'Haití. II

-(130)- VI. Convenio de 8 agosto 1984, [sobre renuncia al privilegio de presentación] ....III

\section{HUNGRÍA}

-(131)- I. Acta [abrogado] con Protocolo de 15 septiembre 1964, entre encargados de la

Santa Sede y el Gobierno de Hungría (Comunicado) . II

-(132)- II. Acuerdo [abrogado] de 23 enero 1969, relativo al nombramiento de Obispos y Administradores Apostólicos (remisión) II

-(133)- III. Acuerdo [abrogado] de 11 enero 1975, (remisión). II

-(134)- IV. Acuerdo de 9 febrero 1990 III

-(135)- V. Acuerdo de 10 enero 1994, sobre la asistencia Religiosa a las Fuerzas Armadas y a la 
Policía de Fronteras

-(136)- VI. Acuerdo de 20 junio 1997, sobre la financiación de las actividades de servicio público ("de la vida de fe"), desarrolladas en Hungría por la Iglesia Católica y de Otras estrictamente religiosas, y sobre algunas cuestiones de naturaleza patrimonial IV

- Protocolo Adicional ...IV

\section{ISRAEL}

-(137)- I. Acuerdo Básico de 30 diciembre 1993 ...................................................................III

Protocolo Adicional de 30 diciembre 1993 ...........................................................III

-(138)- II. Convenio de 10 noviembre 1997, para la ejecución del art. $3 \S 3$ del Acuerdo Básico (sobre la personalidad jurídica)

\section{ITALIA}

-(139)- I. Tratado de 11 febrero 1929 II

-(140)- II. Convenio financiero de 11 febrero 1929 ..............................................................

-(141)- III. Concordato de 11 de febrero de 1929 .............................................................. II

-(142)- IV. Acuerdos con Italia sobre el Tratado y el Concordato (remisiones) ................ II

1. Acuerdo de 2 septiembre 1931, concerniente a la Acción Católica Italiana .......II

2. Convenio de 29 mayo 1932, relativo a la veneranda Arca de San Antonio de Padua

3. Acuerdos de 6 septiembre 1932, 26 diciembre 1935, 6 agosto 1938 y 14 marzo 1942 para la prórroga del término establecido en el artículo 29 letra f, del Concordato

4. Protocolo de 6 septiembre 1932, para la ejecución del artículo X del

Tratado de Letrán

5. Canje de Notas de 25/30 mayo 1937, sobre el traspaso de los privilegios de extraterritorialidad del antiguo palacio de los Convertendi al reconstruido en la "via della Conciliazone". II

6. Canje de Notas de 30 julio/2 agosto 1938, sobre la aplicabilidad de los 
artículos 19 y 20 del Concordato al titular de la Archidiócesis de Roda II

7. Convenio de 13 junio 1939, para la aplicación del artículo 29, letra g. del

Concordato de 11 febrero de 1929 y para la determinación de otras relaciones concernientes a las Iglesias y Capillas palatinas así como el clero respectivo II

8. Documento adicional de 18/19 mayo 1942, al Protocolo del 6 de septiembre de 1932 para la ejecución del artículo X del Tratado Lateranense, relativo a los dos Portadores de la Rosa de Oro II

9. Canje de Notas de 16 marzo/16 abril 1945, sobre el traspaso de los privilegios de extraterritorialidad a las áreas cedidas por permuta a la Santa Sede, por el Ayuntamiento de Roma en las zonas de San Pablo II

10. Canje de Notas de 2 /21 agosto 1946, relativo a la modificación de fórmula del juramento de los Obispos italianos sancionada en el artículo

29 del Concordato II

Canje de Notas de 31 diciembre 1946/26 septiembre 1947, sobre

la nueva fórmula de las Preces propiciatorias previstas

en el artículo 12 del Concordato II

11. Acuerdo de 24 abril 1948, para una nueva delimitación de la zona extraterritorial constituida por las Villas pontificias en Castel Gandolfo-Albano Laziale.

12. Canje de Notas de 17/21 noviembre 1949, relativo a extensión de los privilegios diplomáticos al Secretario de Embajada. II

13. Canje de Notas de 14/18 julio 1951, relativo al nuevo Estatuto de la Iglesia italiana de Nuestra Señora de Loreto en Lisboa II

14. Acuerdo relativo de 8 octubre 1951, a las instalaciones del

Radio-Vaticano en Santa María de Galería y en Castel Romano II

15. Canje de Notas de 16 diciembre 1955, sobre exención de impuestos 
a favor de los Representantes diplomáticos de ciudadanía italiana que se hallan acreditados por terceros Estados ante la Santa Sede. II

16. Acuerdo de 7 julio 1964, sobre modificación de algunas

circunscripciones diocesanas y sobre la erección de la diócesis

de Bolzano-Bressanone

V. Convenios con Italia:

-(143)- 1. Acuerdo de 18 febrero 1984, que aporta modificaciones al Concordato

Lateranense..... III

-(144)- 2. Protocolo Adicional de 18 febrero 1984 III

3. Protocolo de 15 noviembre 1984, de aprobación de las normas para la regulación de la materia mencionada en el art. 7 n. 6 del Acuerdo que aporta modificaciones al Concordato Lateranense III

4. Anexo: Canje de Notas de 15 noviembre 1984 ................................................... III

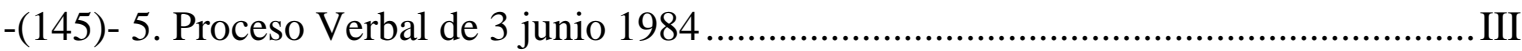

6. Normas sobre entidades y bienes eclesiales: [omissis] III

-(146)- VI. Decreto de 3 junio 1985, normas sobre las entidades y bienes eclesiásticos en Italia y sobre la revisión de los compromisos financieros del estado italiano y de las intervenciones del mismo en la gestión patrimonial de las entidades eclesiásticas IV

-(147)- VII. Canje de Notas de 23 diciembre 1985, sobre Nombramientos eclesiásticos ..IV

-(148)- VIII. Canje de Notas de 23 diciembre 1985, sobre festividades religiosas. IV

-(149)- IX. Canje de Notas de 25 enero 1994, sobre reconocimiento de títulos académicos

-(150)- X. Acuerdo de 15 febrero de 1995, para regular las relaciones entre el Hospital pediátrico "Niño Jesús” y el Servicio Sanitario Nacional. IV

-(151)- XI. Canje de Notas de 10/30 abril 1998, con los Anejos I y II constitutivos de una Convención declarativa y ejecutiva del Acuerdo de 28 febrero de 1984 IV

-(152)- XII. Canje de Notas de 13 noviembre/14 diciembre 1999, constitutivo de Convención Técnica. IV

-(153)- XIII. Convención, de 16-6-2000, sobre la Seguridad

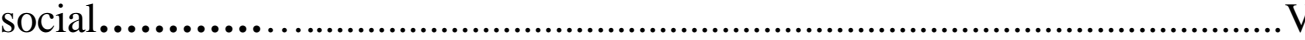

-(154)- XIIV Canje de Notas, de 26-7-2006..............................V 


\section{KAZAJSTÁN}

-(155)- Acuerdo de 24 septiembre 1998, sobre mutuas relaciones IV

\section{LETONIA}

-(156)- Acuerdo [general] de 8 noviembre 2000 IV

\section{LIGA DE ESTADOS ÁRABES}

-(157)- Memorandum de Entendimiento, de 23 de abril 2009

\section{LITUANIA}

-(158)- I. Acuerdo de 5 mayo 2000, concerniente a los aspectos jurídicos de las relaciones entre la Iglesia Católica y el Estado IV

-(159)- II. Acuerdo de 5 mayo 2000, sobre cooperación en Educación y Cultura IV -(160)- III. Acuerdo de 5 de mayo de 2000, sobre la Asistencia pastoral de los católicos que sirven en el Ejército... IV

-(161)- IV. Acuerdo 14 de junio de 2012 $V I$

\section{MALTA}

-(162)- I.1. Acuerdo de 26 septiembre 1988, sobre la incorporación de la Facultad de

Teología a la Universidad de Malta III

I.2.Anexo de 26 septiembre 1988, relativo al Artículo 3 del Acuerdo [precedente] III -(163 )- II.1 Acuerdo de 16 noviembre 1989, para mejor ordenar la instrucción y la educación religiosa católica en las escuelas III

-(164)- II.2Acuerdo de 16 noviembre 1989, entre el Ministro de Educación y el

Presidente de la Conferencia Episcopal de Malta relativo a los Modos regulación sobre la instrucción y la educación católica III

-(165)- II.3.Protocolo adicional de 18 febrero 2003, sobre la instrucción y la educación católica. IV

-(166- III. Acuerdo de 28 noviembre 1991, sobre bienes de la Iglesia temporales 
relativo al traspaso al Estado de la propiedad inmueble de la Iglesia Católica no utilizada para fines pastorales y sobre la determinación de ciertos supuestos pertenecientes a las relaciones entre la Iglesia y el Estado que contemplan materias patrimoniales III

-(167)- IV. Convenio de 28 noviembre 1991, sobre los colegios de la Iglesia. III

-(168)- V.1.Acuerdo de 3 febrero 1993, sobre el reconocimiento de los efectos civiles del matrimonio canónico y de las decisiones de las autoridades eclesiásticas y de los tribunales sobre los mismos matrimonios [CR 25 III 1995] III

-(169)- V.2 bis. Protocolo [primero] de aplicación de 3 febrero 1993. III

-(170)- V.3. Protocolo adicional segundo de 6 enero 1995 IV

-(171)- V.3. Protocolo Adicional de 18 de febrero de 2oo3, para mejorar la instrucción y la educación religiosa en las escuelas estatales IV

\section{MARRUECOS}

(172)- Canje de Notas Diplomáticas de 30 diciembre 1983/5 febrero 1984, sobre el Estatuto de la Iglesia Católica en Marruecos

\section{MONACO}

-(173)- I. Letras Apostólicas de León XIII, Quemadmodum de 15 marzo 1887, creando la Diócesis de Mónaco II

-(174)- II. Convenio, de 25-7-1981 III

\section{MONTENEGRO}

-(175)- Acuerdo de base, de 24-6-2011 V

\section{MOZAMBIQUE}

-(176)- Acuerdo de 8 de diciembre de 2011 


\section{ORGANIZACIÓN DE LA UNIDAD DE AFRICA (O.U.A.)}

-(177)- Acuerdo de cooperación, de 19 octubre 2000. IV

\section{PALESTINA (O.L.P.)}

-(178)- Acuerdo básico de 15 febrero 2000, entre la Santa Sede y la Organización para la Liberación de Palestina (O.L.P.)

\section{PARAGUAY}

-(179)- I. Convenio, de 26-11-1960, sobre la erección de Vicariato Castrense. II

Protocolo Adicional de 26-11-1960 II

-(180)- II. Convenio, de 24-12-2002, sobre asistencia religiosa a as Fuerzas Armadas....V 239

\section{PERÚ}

-(181)- I. Letras apostólicas de 5 marzo 1875, Praeclara inter beneficia :concesión del derecho de patronato ...II

-(182)- II. Acuerdo [general] de 19 julio 1980. II

\section{POLONIA}

-(183)- I. Acuerdo [abrogado] de 8 julio 1974, relativo a la institucionalización de grupos permanentes de trabajo (remisión)..... .II

-(184)- II.1. Concordato de 28 julio 1993 IV

-(185)- II.2.Concierto de 1 julio 1999 [omissis], entre el gobierno de la República de Polonia y la Conferencia Episcopal Polaca, sobre el estatuto jurídico de los centros superiores instituidos y dirigidos por la Iglesia Católica -comprendidos aquí las Universidades, las facultades autónomas y los seminarios mayores eclesiásticos- y sobre las modalidades y términos del reconocimiento, por parte del Estado, de los grados y títulos académicos conferidos por éstos] IV

\section{PORTUGAL}

-(186)- I. Concordato de 7 mayo 1940 [abrogado]

-(187)- II. Acuerdo Misional de 7 mayo 1940... [abrogado] 
-Canje de Notas, de 7 mayo 1940, completando el Acuerdo Misional (remisión)

-(188)- III. Acuerdo de 18 julio 1950... [abrogado]

-(189)- IV. Acuerdo de 15 febrero 1975, para cambiar el artículo XXIV del Concordato de 7 mayo de $1940 \ldots$ [abrogado]

-(190)- V. Concordato, de 18-5-2004

\section{REPÚBLICA DOMINICANA}

-(191)- I. Concordato de 16 junio 1954

Protocolo Final de 16 junio 1954

-(192)- II. Acuerdo de 21 enero 1958

Anejo: Reglamento

-(193)- III. Protocolo Adicional de 11 mayo 1990, sobre el Ordinariato castrense

\section{SAN MARINO}

-(194)- I. Acuerdo de 11 julio 1989, sobre el reconocimiento de las fiestas religiosas

-(195)- II. Acuerdo [general] de 2 febrero 1992

\section{SUECIA}

-(196)- Canje de Notas de 24 de noviembre 2001 [sobre personalidad de la Iglesia Católica Romana]

\section{SUIZA}

-(197)- I. Convención de 23 octubre 1870, sobre la anexión de las dos parroquias de Poschiavo y Brusio al Obispado de Coira 
-(198)- II. Convenio de 1 septiembre 1884, sobre la administración regular de la diócesis de Basilea

-(199)- III. Convenio de 3 julio 1968, sobre la separación de la Administración Apostólica del Cantón Ticino de la diócesis de Basilea y la erección de la misma diócesis

-(200)- IV. Convenio Adicional, de 2 mayo 1978, sobre la diócesis de Basilea

\section{ARGOVIA Y TURGOVIA}

-(201)- I. Convenio de 2 diciembre 1828, sobre la incorporación de los Cantones de Argovia y Turgovia al Obispado de Basilea

\section{BERNA}

-(202)- I. Convenio de 26 marzo 1828, sobre la reorganización y nueva circunscripción del Obispado de Basilea

-(203)- II. Convenio de 11 julio 1864, relativo a la incorporación de la parte antigua del

Cantón de Berna a la diócesis de Basilea

\section{FRIBURGO}

-(204)- I. Intercambio de Notas diplomáticas de 26 junio/25 julio 1924

\section{SAN GALL}

-(205)- I. Convenio de 7 noviembre 1845, sobre la reorganización del Obispado de San Gall

\section{LUCERNA}

-(206)- I. Acuerdo de 11 junio 1926, sobre los privilegios en la provisión de oficios y beneficios eclesiásticos

TICINO

-(207)- I. Convenio de 23 septiembre 1884, sobre la Administración Apostólica del

Cantón Ticino

\section{TÚNEZ}

-(208)- Convenio (Modus vivendi) [general] de 27 junio 1964

Protocolo Adicional de 27 junio 1964

Canje de Notas de 27 junio 1964 


\section{UNIÓN EUROPEA}

-(209)- Convención monetaria, de 17-12-2009

\section{VENEZUELA}

-(210)- I. Convenio [general] de 6 marzo 1964

-(211)- II. Acuerdo de 24 noviembre 1994, sobre el ordinariato castrense

\section{VIETNAM}

-(212)- Canje de Notas de 1990/1995 [sobre nombramientos episcopales]

\section{RELACIÓN CRONOLÓGICA de los 220 Concordatos y/o Acuerdos vigentes.}

- Si algún Concordato/ y/o Acuerdo dejara de estar vigente (por más que hubiera sido publicado en uno de nuestros 5 tomos), se indicará oportunamente que está abrogado. No obstante se recogen Concordatos/ y/o Acuerdos, por más que se digan abrogados, en cuanto que siguen teniendo influencia o vienen citados/remitidos en Concordatos y/o Acuerdos posteriores.

- con dos ** van señalados los expresamente titulados "Concordatos".

- con un * los Acuerdos (Convenios, Protocolos, Canjes) que contengan una regulación general

- Para la Cronología de los Concordatos seguimos el Enchiridion dei Concordati, Due secoli di Storia dei rapportei Chies-Stato (Bologna,EDB 2003)

++PÍO VII (1800-1823)

-- del s. XIX

180115 VII **Francia, Concordato

++LEÓN XII (1823.1829)

18282 XII Suiza: Argovia y Turgovia, Obispado de Basilea

182826 III Suiza: Berna, Diócesis de Basilea 
++GREGORIO XVI (1831-1846)

18457 XI Suiza: San Gall, Obispado

++PÍO IX (1846-1878)

$186028 \mathrm{II} * *$ Haití, Concordato

18612 III Haití, Circunscripciones diocesanas

186217 VI Haití, Dotación

186411 VII Suiza: Berna, Diócesis de Basilea

187023 X Suiza, Diócesis de Coira

18755 III $\quad$ Perú, Bula concordada

++LEÓN XIII (1878-1903)

1884 1 IX Suiza, Diócesis de Basilea

188423 IX Suiza: Ticino, Administración Apostólica de. Ticino

188715 III Mónaco, Letras Apostólicas de León XIII

\section{-- del siglo XX}

+1902 5 XII Francia, Facultad de Teología

++PÍO X (1903-1914)

++BENEDICTO XV (1914 -1922)

1921 mayo Francia, Nombramientos episcopales

++PÍO XI (1922-1939)

1923 7/13 V Francia, Canje, Estatuto asociaciones diocesanas.

1923 16/17 VI Francia, Facultad de. Teología

192429 III **Baviera, Concordato. 
192426 VI Suiza: Friburgo, Notas

192611 VII Suiza: Lucerna, Administración de Ticino

19264 XII Francia, Honores litúrgicos

192911 II **Italia, Concordato.

192914 VI ** Prusia, Concordato.

1931 Italia, Varios convenios.

19324 I Anhalt, Escuelas

$193212 \mathrm{X} \quad * *$ Baden, Concordato

$19335 \mathrm{VI} * *$ Austria, Concordato

193320 VII ***emania, Concordato.

193724 VII *Ecuador, Modus vivendi

PíO XII (1939-1958)

$\begin{array}{llll}1940 & 7 \text { V } & \text { **Portugal, Concordato. (abrogado) } \\ 1940 & 7 \text { V } & \text { Portugal, Misiones (abrogado) } \\ 1940 & 25 \text { I } & \text { Haití, Bienes. } \\ 1941 & 7 \text { VI } & \text { España, Privilegio de Presentación [abrogado] } \\ 1946 & 6 \text { VII } & \text { España, Dotación [abrogado] } \\ 1946 & 8 \text { VII } & \text { España, Seminarios [abrogado] } \\ 1947 & 7 \text { IV } & \text { España, Tribunal de la Rota } \\ 1950 & 18 \text { VII } & \text { Portugal, India (misiones) [abrogado] } \\ 1950 & 5 \text { VIII } & \text { España, Castrense [abrogado] } \\ 1952 & 20 \text { IX/28 III } & \text { Filipinas, Castrense } \\ 1953 & 5 \text { VIII } & \text { **España, Concordato_[abrogado] } \\ 1953 & 27 \text { VIII } & \text { España, Bula [abrogado] } \\ 1954 & 16 \text { VI } & * * \text { Rep. Dominicana, Concordato. } \\ 1956 & 19 \text { XII } & \text { Renania del Norte - Westfalia. }\end{array}$


195728 VI Argentina, Castrense

19574 XII Bolivia, Misiones

195821 I Rep. Dominicana, Castrense

195828 XI Bolivia, Castrense

1958 29/30 VIII Baviera, Acuerdos Adicionales y Administrativo.

196023 VI Austria, Administración de Burgenland

196023 VI Austria, Dotación

196026 XI Paraguay Castrense

19625 IV España, Universidades

19629 VII Austria, Escuelas de la Iglesia

++PABLO VI (1963-1978)

19646 III * Venezuela, Convenio General

196427 VI *Túnez, Modus vivendi-General

19647 VII $\quad$ Austria, Administración de. Innsbruck

196415 IX Hungría, Comunicado

196526 II ***Baja Sajonia, Concordato.

196531 VII Alemania, Castrense (remisión)

196622 II Alemania, Interpretación

$196625 \mathrm{VI} \quad$ *Yugoslavia [abrogado]

196615 VIII Haití, Nombramientos

19662 IX Baviera, Teología

$196610 \mathrm{X} \quad *$ Argentina, Acuerdo general

1967 20/29 XII Renania del Norte - Westfalia, (remisión)

196811 III El Salvador, Castrense

19689 IV Sarre, Teología

196824 VII Suiza: Administración de.Ticino 
19687 X Austria, Diócesis Feldkirch

196923 I Hungría, Nombramientos episcopales (abrogado)

1969 21/22 IV Renania del Norte - Westfalia, Maestros

196929 IV Renania-Platinado, Modificación del Conc

196929 IX Austria, Dotación

196912 XI Sarre, Maestros

197027 IX Baviera, Teología

19718 III Austria, Escuelas

19729 I Austria, Escuelas

197327 II Checoslovaquia, Nombramientos episcopales (remisión)

197315 V Renania-Platinado, Escuelas

197321 V Baja Sajonia, Modificación del Concordato.

197312 VII **Colombia, Concordato

197425 V Francia, Pedagogía religiosa

19748 VII Polonia, Grupos de trabajo (abrogado)

19744 IX Baviera, Modificación del Concordato.

197511 I Hungría, Acuerdo (abrogado)

197515 II Portugal, Matrimonio

197521 II Sarre, Escuelas de la Iglesia

19752 VII Colombia, Declaración

19769 I Austria, Dotación

197628 VII España, Privilegios

19782 V $\quad$ Suiza, Diócesis de Basilea

19783 VIII Ecuador, Castrense

19787 VIII Baviera, Modificación del Concordato 
19793 I España, Asuntos jurídicos

19793 I España, Castrense

19793 I España, Educación

19793 I España, Asuntos económicos

$198010 X \quad$ España, Tributos

198026 XI *Perú, Acuerdo general

198125 VII Mónaco, Arzobispado

1983/84 30 XII y 5 II *Marruecos, Estatuto Iglesia

198418 II/15 XI *Italia:

*[1] Modificación del Concordato.

[2] Protocolo Adicional

[3] Protocolo de aprobación de las normas

[4] Anexo

[5] Proceso Verbal

[6]. Normas sobre entidades y bienes

198426 III Renania, Enseñanza Religión

19848 VIII Haití, Nombramientos episcopales

198512 II Sarre, Enseñanza religión

19853 VI Italia, Decreto ... normas...patrimonio

198523 XII Italia, Nombramientos eclesiásticos

19861 XII Bolivia, Castrense

19888 VI Baviera Modificaciones del Concordato.

198826 IX Malta, Facultad de Teología

19895 VII Camerún, Instituto Católico

198914 VIII Costa de Marfil, Estaciones de radiodifusión

198910 X Austria, Dotación.

198916 XI Malta, Educación religiosa. 
$1990 \quad$ Vietnam, Nombramientos

1990 10/16 I Alemania, Castrense

19909 II Hungría, General

$199011 \mathrm{~V}$ República dominicana, Ordinariato castrense

199023 X Brasil, Castrense

19913 II Malta, Matrimonio

199128 XI Malta Propiedad inmueble

199128 XI Malta, Colegios de la Iglesia.

199221 IV Argentina, Castrense

199220 V Costa de Marfil, Yamusucro

199220 XI Colombia, Modificación del Concordato

199211 XII *San Marino, Acuerdo General.

$1993 \quad 3$ II Malta, Matrimonio

199328 VII **Polonia, Concordato

199329 X Baja Sajonia, Modificación del Concordato

199329 X Baja Sajonia, Interpretación.

199329 X Baja Sajonia, Protocolo final

199330 XII *Israel, Acuerdo básico

199410 I Hungría, Castrense

199425 I Italia, Títulos académicos

199413 IV Sajonia-Anhalt, Brandeburgo y Sajonia, Diócesis de Magdeburgo

199413 IV Brandeburgo, Diócesis de Görlitz

199413 IV Turingia, Diócesis de Erfurt

199422 IX Hamburgo, Arzobispado y Provincia Eclesiástica de Hamburgo

199421 X Venezuela, Castresne

199421 XII España, Tierra Santa

19956 I Malta, Matrimonio 


\begin{tabular}{|c|c|c|}
\hline 1995 & $26 \mathrm{I}$ & Malta, Facultad de Teología \\
\hline 1995 & 15 II & Italia, Hospital "Niño Jesús" \\
\hline 1995 & $9 \mathrm{~V}$ & Vietnam, Nombramientos \\
\hline 1995 & $21 \mathrm{XI}$ & Austria, Cuestiones patrimoniales \\
\hline 1995 & 17 VIII & Camerún, Instituto Católico \\
\hline 1996 & $2 \mathrm{IV}$ & *Sajonia, General \\
\hline 1996 & 19 XII & *Croacia, Cuestiones jurídicas \\
\hline 1996 & 19 XII & Croacia, Educación y cultura \\
\hline 1996 & 19 XII & Croacia, Ordinariato Castrense \\
\hline 1997 & 10/30 IV & Italia, Convención declarativa y ejecutiva \\
\hline 1997 & $11 \mathrm{VI}$ & *Turingia, Acuerdo general \\
\hline 1997 & $20 \mathrm{VI}$ & Hungría, Entes religiosos, patrimonio y cultura \\
\hline$* 1997$ & $718 X$ & *Mecklenburgo-Pomerania, Acuerdo general \\
\hline 1997 & $10 \mathrm{XI}$ & Israel, Personalidad jurídica \\
\hline 1997 & $12 \mathrm{XII}$ & *Gabón, Acuerdo Marco \\
\hline 1998 & $15 \mathrm{I}$ & *Sajonia-Anhalt, Acuerdo general \\
\hline 1998 & $24 \mathrm{IX}$ & Kazajstán, Mutuas relaciones \\
\hline 1998 & $9 x$ & Croacia, Cuestiones económicas \\
\hline 19982 & 23-XII/99 15 II & *Estonia, Acuerdo general \\
\hline 1999 & $21 \mathrm{I}$ & Francia, Trinità dei Monti \\
\hline 1999 & $12 \mathrm{II}$ & *Estonia, Canje, Acuerdo general \\
\hline 2000 & $15 \mathrm{I}$ & *Organización Liberación Palestina, Acuerdo básico \\
\hline 2000 & $5 \mathrm{~V}$ & Lituania, Educación y cultura \\
\hline 2000 & $5 \mathrm{~V}$ & *Lituania, Relaciones jurídicas \\
\hline 2000 & $5 \mathrm{~V}$ & Lituania, Ordinariato castrense \\
\hline 2000 & $16 \mathrm{VI}$ & Italia, Convenio sobre Seguridad social \\
\hline 2000 & $19 \mathrm{X}$ & *Unión Africana, Acuerdo general \\
\hline
\end{tabular}




$$
\begin{array}{lll}
2000 & 8 \text { XI } & \text { *Letonia, Acuerdo general } \\
2000 & 24 \text { XI } & \text { *Eslovaquia, Acuerdo base } \\
2000 & 13 \text { XI/14 XIIR.2004 Italia, Convención técnica. }
\end{array}
$$

\section{-- del s. XXI}

$\begin{array}{llll}2001 & 26 \text { VII } & \text { Gabón, } & \text { Enseñanza católica } \\ 2001 & 19 \text { IX } & \text { Sarre, } & \text { Escuelas católica } \\ 2001 & 24 \text { XI } & \text { Suecia, } & \text { Personalidad jurídica de la Iglesia } \\ 2001 & 14 \text { XII } & \text { Eslovenia, Cuestiones jurídicas } \\ 2001 & 12-\text { XI y 4-XII } & \text { Francia. } & \text { Obras Misioneras } \\ 2002 & 23-\text { III } & \text { *Albania Acuerdo General } \\ 2002 & 25 \text { VII } & \text { República } & \text { Checa, Acuerdo General [no-aprobado] } \\ 2002 & 16-\text { XII } & \text { Turingia, Facultad de Teología de Erfurt } \\ 2002 & 24 \text { XII } & \text { Paraguay Castrense } \\ 2003 & 18-\text { II } & \text { Malta } & \text { Educación } \\ 2003 & 21 \text { XI } & \text { *Bremen } & \text { General } \\ 2003 & 12 \text { XI } & \text { *Brandeburgo } & \text { General }\end{array}$

++BENEDICTO XVI (2005 19-IV----28-II-2013)

$200418 \mathrm{~V} \quad * *$ Portugal, $\quad$ Concordato

$2004 \quad 13 \mathrm{~V} \quad$ Eslovaquia Educación

200512 VII Francia Trinità in Monte

200529 XI *Hamburgo Acuerdo General.

2006 16. I Italia Seguridad social.

2006 28-IV Bosnia-Herzegovina Acuerdo general

2006 21/22-XII España Impuestos.

2007 19-I Baviera Protocolo Adicional

$2007 \quad 17$ IV Filipinas Acuerdo Cultural 


\begin{tabular}{|c|c|c|c|c|}
\hline 2007 & $13 \mathrm{XII}$ & Albania & \multicolumn{2}{|c|}{ Econom-ia- Tributación } \\
\hline 2008 & $17 \mathrm{III}$ & *Andorra & \multicolumn{2}{|c|}{ Acuerdo General } \\
\hline 2008 & $13 \mathrm{XI}$ & *Brasil & \multicolumn{2}{|c|}{ Acuerdo General } \\
\hline 2008 & $18-\mathrm{XI}$ & Francia, & \multicolumn{2}{|c|}{ Títulos eclesiásticos. } \\
\hline 2009 & $12 \mathrm{I}$ & \multicolumn{2}{|c|}{ *Schleswig-Hostein } & Acuerdo General \\
\hline 2009 & $23 \mathrm{IV}$ & \multicolumn{2}{|c|}{ Liga de Estados Árabes } & s Memorandum \\
\hline 2009 & $20 \mathrm{VIII}$ & \multicolumn{2}{|l|}{ Bolivia } & Unidades educativas \\
\hline 2009 & $10 \mathrm{III}$ & \multicolumn{2}{|l|}{ Austria } & Dotación \\
\hline 2009 & $12 \mathrm{I}$ & \multicolumn{2}{|c|}{ *Schleswig-Holstein } & Acuerdo General \\
\hline 2009 & 17-XII & \multicolumn{2}{|c|}{ Unión Europea } & Convención monetaria \\
\hline 2010 & $6 \mathrm{IV}$ & \multicolumn{2}{|l|}{ Baja Sajonia } & Educación \\
\hline 2010 & $18 \mathrm{~V}$ & \multicolumn{2}{|l|}{ Hamburgo } & Teología \\
\hline 2011 & $24 \mathrm{VI}$ & \multicolumn{2}{|l|}{ *Montenegro } & Acuerdo de base \\
\hline 2011 & $12 \mathrm{XI}$ & \multicolumn{2}{|l|}{ *Brandeburgo } & Acuerdo General \\
\hline 2012 & 15-VII & \multicolumn{2}{|l|}{ Lituania } & Enseñanza: Títulos \\
\hline 2012 & 18-VII, & \multicolumn{2}{|c|}{ Unión Europea } & Convención monetaria \\
\hline 2012 & $6 \mathrm{XI}$ & \multicolumn{2}{|c|}{ *Guinea Ecuatorial } & Acuerdo General \\
\hline 2012 & 17 XII, & \multicolumn{2}{|c|}{ China/Taiwán } & Acuerdo cultural \\
\hline
\end{tabular}

++FRANCISCO I (2013-III .....)

$2013611 \quad$ Chad Acuerdo General 